\title{
Transatlantica
}

Revue d'études américaines. American Studies Journal

\section{Richard Godden, William Faulkner, An Economy of Complex Words}

Princeton : Princeton University Press, 2007

\section{Frédérique Spill}

\section{(2) OpenEdition}

\section{Journals}

Édition électronique

URL : http://journals.openedition.org/transatlantica/2032

DOI : 10.4000/transatlantica.2032

ISSN : 1765-2766

Éditeur

AFEA

\section{Référence électronique}

Frédérique Spill, «Richard Godden, William Faulkner, An Economy of Complex Words », Transatlantica [En ligne], 2 | 2007, mis en ligne le 14 mai 2008, consulté le 29 avril 2021. URL : http://

journals.openedition.org/transatlantica/2032 ; DOI : https://doi.org/10.4000/transatlantica.2032

Ce document a été généré automatiquement le 29 avril 2021.

\section{c) (i) $\Theta$}

Transatlantica - Revue d'études américaines est mis à disposition selon les termes de la licence Creative Commons Attribution - Pas d'Utilisation Commerciale - Pas de Modification 4.0 International. 


\section{Richard Godden, William Faulkner, An Economy of Complex Words}

Princeton : Princeton University Press, 2007

Frédérique Spill

1 En guise de préambule, Richard Godden s'empresse de préciser que le lien entre économie et langage qui est stipulé dans le titre de son dernier ouvrage critique sur l'œuvre de William Faulkner ne doit pas être compris de manière métaphorique : c'est bien en fonction de leur rapport avec un contexte économique et social spécifique que sont analysées les complexités de l'écriture faulknérienne. Sa démonstration repose sur trois présupposés qui sont clairement exposés dans son introduction: les relations économiques sont des relations sociales travesties; les relations sociales influencent la possibilité même d'un récit; enfin, les structures économiques sont une source génératrice de formes fictives.

2 Godden prend soin de dresser un bref état des lieux économique du Sud des États-Unis, au moment où les réformes agricoles mises en place par la politique du New Deal (1933-1938) déclenchent une révolution sans précédent dans l'organisation du travail dans les campagnes. En effet, les propriétaires qui dépendaient jusque-là de la maind'œuvre noire deviennent subitement dépendants de capitaux issus du Nord, tandis que les métayers se retrouvent précarisés par les effets pernicieux des aides financières : "Land as "sweat" gave way to land as "capital" » (2).

3 La lecture proposée par Richard Godden, qui choisit ici de se concentrer exclusivement sur trois œuvres relativement tardives de Faulkner - The Hamlet (1940), Go Down, Moses (1942), A Fable (1954) - se focalise en grande partie sur les formes de la perte et du deuil subséquentes à cette modernisation économique et sociale: « My study seeks to trace the demise and reformation of a class by anatomizing the varieties of mourning exhibited by Faulkner's white landowners as they grasped the consequences of modernity in the New Deal's reconstruction of their depressed region » (4). Il considère les trois romans qui attirent plus particulièrement son attention comme les produits d'une modernisation différée et de ses conséquences. 
4 Les deux premiers chapitres du volume sont consacrés à The Hamlet. L'originalité du premier chapitre, intitulé "Earthing The Hamlet », consiste à déconstruire l'image à laquelle la critique tend presque systématiquement à identifier le personnage de Flem Snopes: "Flem may be misread if he is too readily allegorized as the agent of something as generic as "capital" »(18). Plutôt que de voir en Flem Snopes, aussi taciturne soit-il, un agent stratégique et tacticien du capital, Godden le considère essentiellement comme un individu chanceux. Sa démonstration, qui s'amorce sur une interprétation de l'accoutrement de Flem Snopes, se construit sur une analyse minutieuse du point de vue de Ratliff, dont les pensées se mêlent souvent indistinctement à celles du narrateur à la troisième personne - «the narrator in Ratliffian mode » (32). Alors que Ratliff, colporteur d'histoires et de machines à coudre, emporte d'ordinaire les suffrages de la critique, Godden s'attache à montrer combien la manière dont il perçoit Flem Snopes manque d'objectivité : «Ratliff's perception of Flem needs to be recognized as partial, interested, and class-based»(21). Godden interprète Ratliff, qui met précisément un point d'honneur à se présenter comme la parfaite antithèse de Flem, comme une figure de double et leurs trajectoires respectives comme parallèles : tous deux sont en effet issus de milieux modestes, tous deux ont quitté les champs pour se lancer dans le commerce; mais c'est là que leurs parcours diffèrent.

5 La démonstration de Godden s'apparente en quelque sorte à une lente érosion de la bienveillance apparente de Ratliff : le succès de son affaire, insiste-t-il, repose sur la même logique d'endettement qui assure la pérennité de riches propriétaires comme Will Varner en lui asservissant ses clients - " as "usurer", "owner of most of the good land in the country," and mortgagee "on most of the rest" »(19). Pour sa part, Flem Snopes (dont le portrait résulte, démontre-t-il, d'une construction fictive dont Ratliff se sert comme d'un exutoire et d'un prétexte pour échapper à sa propre culpabilité et aux traitrises engendrées par sa propre modernisation) apparaît paradoxalement grandi par sa cohérence avec la tradition familiale radicale incarnée par son père $A b(n e r)$ Snopes, dont l'imaginaire collectif se souvient comme d'un incendiaire de granges appartenant à la classe des possédants. La nouvelle «Barn Burning», parue en 1938, sert ici constamment de palimpseste : Flem, conclut Godden, est le gardien du «feu » paternel.

6 Dans le second chapitre intitulé "Comparative Cows: Reading The Hamlet for Its Residues ", Godden centre son analyse sur la fonction symbolique des vaches autour desquelles se greffent plusieurs intrigues secondaires dans The Hamlet. L'histoire de Mink Snopes et celle de Ike Snopes présentent en effet de nombreux points communs : "they share significant connections, each being bovine in focus, and implicitly appealing to an archaic social form. That Mink's bull has the political properties of Ab's fire should not distract from its species affinity with Ike's cow. Bull and cow are linked not simply as disputed stock, but as beasts through which a Snopes seeks access to use rights in the land » (51).

7 La première vache qui attire l'attention de Godden est donc celle qui est à l'origine du litige opposant Houston à Mink Snopes. Ce litige se dénouera de manière dramatique par le meurtre de Houston, représentant de la classe des propriétaires, par Mink. Godden interprète le personnage de Mink Snopes comme une sorte d'emblème de la dépossession. Ainsi propose-t-il de lire ce meurtre à la manière d'un geste politique radical, comme l'expression extrême de la lutte des classes menée, de façon générale, 
par les Snopes - mais encore faut-il ajouter : chacun à leur manière. Godden précise aussitôt sa pensée, qui emprunte aux réflexions de Hegel sur la dialectique du maître et de l'esclave. Dans le cas de Mink, cette lutte s'apparente, d'après lui, à une rébellion esclavagiste: «to read attentively is to learn that the residual meaning of the events triggered by the bull derive from archaic structures of feeling associated not with white landlords and white tenants, but with masters and slaves. In effect, Mink's disputed bull prompts a "pre-political act" of populist resentment whose deep structure is that of a slave revolt » (43). D'après Godden, les structures qui déterminent la relation du maître et de l'esclave opèrent d'ailleurs à tous les niveaux de l'existence de Mink: elles constituent un cadre infrangible qui conditionne même ses échanges sexuels avec la fille de maître, masculine et autoritaire, qui deviendra Mrs Snopes.

8 La seconde vache sur laquelle se construit l'analyse de Godden est celle dont s'éprend l'idiot Ike Snopes. D'après Godden, la vache en question n'est, d'une certaine manière, qu'un prétexte pour permettre à l'idiot d'accéder à son véritable amour, à savoir la terre: "Ike's cow grants him access to his true love-the soil, which both nourishes him and is the object of his passion » (52). Ainsi Godden reprend-il le fil de sa réflexion, amorcée précédemment, sur la longue série de personnages "autochtones " (au sens littéral du terme) qui entretiennent un lien privilégié avec la terre, ce qui le conduit à considérer une troisième et dernière vache dans The Hamlet, à savoir Eula Varner Snopes, héroïne terrienne par excellence : « Faulkner casts the bovine as earth's orifice, not to sexualize the earth but to earth sexuality » (58).

Dans les deux chapitres suivants, Godden s'intéresse essentiellement aux nouvelles de Go Down, Moses qui constituent ce qu'on s'accorde à considérer comme «la trilogie des plantations ", ainsi qu'à "Old People », la quatrième nouvelle du recueil. À l'occasion de son analyse du conflit opposant Zack et Lucas dans "The Fire and the Hearth", Godden poursuit sa réflexion sur l'interdépendance des figures du maître et de l'esclave: "the decision to kill the master amounts to self-murder, hence the symptoms of Lucas's protracted dissolution » (66). Il expose les raisons pour lesquelles le meurtre de Zack par Lucas est de toute éternité condamné à l'échec. D'une manière plus générale, sa réflexion se construit à ce stade autour de trois axes majeurs: la notion de "revenants ", à savoir le retour persistant de formes archaïques déplacées par la restructuration du travail ; le traitement paradoxal chez Faulkner de la notion de migration (Godden remarque en effet que même s'ils effectuent d'incessantes pérégrinations, les travailleurs noirs dans Go Down, Moses ne quittent pas le Sud pour le Nord, se démarquant ainsi d'une tendance historique); enfin, la notion de deuil, comprise à la fois de manière symbolique comme une réaction collective à l'évanouissement de formes ancestrales organisant le travail et de manière littérale comme réponse individuelle à la perte d'un être cher (c'est Rider perdant sa femme Mannie dans "Pantaloon in Black»). Son analyse des figures du deuil emprunte notamment aux écrits psychanalytiques de Nicolas Abraham et Maria Torok sur la notion d'incorporation : "Although sick with grief, Rider cannot mourn since to do so would be to acknowledge the finality of his wife's death. Instead he incorporates her, turning his body into a preservative crypt » (108).

Dans ces chapitres, les nombreux commentaires de textes, précis et détaillés, qui émaillent la prose de Godden s'attachent à mettre en relief la double scission des mots et des choses, et l'ambivalence de la langue que Faulkner a, d'après lui, érigées en stratégie narrative : «Nouns are split and their objects divided by Faulkner's choice of 
free indirect discourse as a prevalent narrative option throughout Go Down, Moses» (69) - ce qu'il appelle ailleurs "la mobilité indirecte libre de Faulkner». Godden insiste également sur la structure essentiellement parataxique de Go Down, Moses : « any coordination of its parts depends upon inferences cast across pauses and changes in narrative direction, instigated by the gaps between the stories themselves »(96). La spécificité de la structure du recueil le conduit à procéder à d'incessantes révisions de ses propres analyses de l'une des nouvelles à la lumière de sa lecture d'une autre nouvelle. Cette méthode lui permet en outre de définir un certain nombre de scènes canoniques (ce qu'il appelle les "ur-scenes»), dont le schéma se répète d'une nouvelle à une autre. C'est ainsi qu'à mi-chemin de son parcours, il décrit dans le même mouvement sa démarche et l'une de ces ur-scenes que chacune des premières nouvelles de Go Down, Moses reproduit en lui apportant à chaque fois quelques variations: "I have been engaged in a formal exercise, fabricating a scene from the recurrent parts of other scenes. The scene features a male pairing on a bed, a missing female, and a mortally wounded white body » (98).

11 C'est la même méthode d'analyse qui est à l'œuvre dans le chapitre rédigé en collaboration avec Noel Polk qui, en prenant pour point de départ l'intérêt que leur témoigne Ike McCaslin, se donne pour objet l'examen des registres du domaine des McCaslin dans "The Bear", le cinquième récit de Go Down, Moses: "the commissary ledgers in which his forebears have recorded the business and personal affairs of the McCaslin family over the course of the plantation's life during the first half of the nineteenth century» (120). Cette plongée dans «The Bear », qui est probablement l'un des textes faulknériens les plus redoutés pour son extrême difficulté, témoigne d'une grande originalité. En vertu de son ingéniosité et de l'audace de certaines de ses conclusions, ce chapitre, qui se lit d'une certaine manière comme une longue enquête policière reposant sur le déchiffrage d'entrées de registres aussi cryptiques que minimalistes, est probablement l'un des plus passionnants du volume.

12 Au-delà des conclusions proposées et bien qu'il ne se revendique pas comme tel, ce chapitre se déploie comme une leçon d'analyse de texte : en effet, par un jeu de miroirs, les auteurs mènent leur investigation, à la fois constituée de micro-lectures minutieuses et d'allers-retours dans le texte et les textes faulknériens, en imitant et en critiquant les méthodes mises en œuvre par Ike dans sa propre analyse des registres. L'analyse des informations concernant l'esclave Percival Brownlee se révèle particulièrement fertile, dans la mesure où elle met en lumière combien le travail d'interprétation de Ike est nécessairement biaisé par le fait qu'il a, de toute éternité, décidé de ses conclusions : son unique but consiste en effet à prouver la perfidie de son grand-père à l'endroit de ses esclaves en déterrant de vieilles et sombres histoires de relations interraciales et d'inceste. Or, à travers leur contre-enquête, qui met en relief un certain nombre d'hésitations et formule par anticipation un certain nombre de réponses aux éventuelles réserves du lecteur, Godden et Polk s'attachent à dépister les différentes interprétations possibles de mêmes événements : « Now we may read what we understand as that which has been forgotten in the ledgers; forgotten because Ike's constructive attention to those ledgers has occluded viable alternatives. Our act of unforgetting seeks to give to Eunice a different death»(142). S'intéressant aux questions d'onomastique et proposant des interprétations très étayées de pronoms cryptiques, Godden et Polk font ainsi ressortir les stratégies de masquage et de démasquage qui sous-tendent le texte de "The Bear». Dans cette perspective, ils montrent que les conclusions hâtives de Ike, soucieux de condamner son grand-père, 
masquent la question de l'ambiguïté des choix sexuels de son propre père : «L.Q.C.'s utter wickedness lies largely within Isaac's narrative gift-one that potentially allows him to displace onto his grandfather his father's acts of homosexual incest and miscegenation » (148).

Les deux derniers chapitres du volume se concentrent sur A Fable, l'un des romans de Faulkner qui a le plus été négligé par la critique. Ces deux chapitres sont donc particulièrement bienvenus. Ils prennent tous deux le parti de se construire autour des figures elliptiques de la judaiité dans le roman. Le premier met en relief le traitement faulknérien de la question de la militarisation: «A Fable, though set during a single week of the Great War, addresses militarization in a more extensive sense »(156) - roman allégorique, A Fable se déploie, au-delà du contexte de la Première Guerre Mondiale, comme une réflexion politique sur des problèmes contemporains. Le second chapitre traite de la perte et des figures empruntées par la mélancolie.

Même si on peut regretter que William Faulkner. An Economy of Complex Words ne s'achève pas sur un chapitre conclusif qui se proposerait de nouer les suggestions et intuitions souvent provocantes et novatrices auxquelles l'ont conduit ses analyses, la dernière étude de Richard Godden sur William Faulkner est d'une grande pertinence. D'une part, elle offre un éclairage inédit sur trois œuvres que l'on trouve peu souvent au centre de l'attention critique. Malgré son parti pris qui consiste à lire l'œuvre de Faulkner à la lumière d'un contexte historique spécifique, il s'agit, d'autre part, d'une étude littéraire au sens fort du terme. En effet, par l'attention à la fois révérencieuse et audacieuse qu'il consacre à la langue de Faulkner (tout en offrant au lecteur la possibilité de suivre dans tous ses soubresauts l'enquête analytique qu'il mène sur le texte et dans le texte), Godden invite le lecteur de Faulkner à poursuivre son propre dialogue avec les intenses complexités de cette écriture singulière. De manière implicite, il lui propose, en outre, une méthode de lecture, au-delà des trois romans dont il est ici question.

INDEX

Thèmes : Recensions

\section{AUTEUR}

FRÉDÉRIQUE SPILL

Université de Picardie, Jules Verne, Amiens, frederiquespill@hotmail.com 